\title{
Different anthropometric adiposity measures and their association with cardiovascular disease risk factors: a meta-analysis
}

\author{
S. B. van Dijk • T. Takken • E. C. Prinsen • H. Wittink
}

Published online: 10 January 2012

(C) The Author(s) 2012. This article is published with open access at Springerlink.com

\begin{abstract}
Objectives To investigate which anthropometric adiposity measure has the strongest association with cardiovascular disease (CVD) risk factors in Caucasian men and women without a history of CVD.

Design Systematic review and meta-analysis.

Methods We searched databases for studies reporting correlations between anthropometric adiposity measures and CVD risk factors in Caucasian subjects without a history of CVD. Body mass index (BMI), waist circumference, waist-to-hip ratio, waist-to-height ratio and body fat percentage were considered the anthropometric adiposity measures. Primary CVD risk factors were: systolic blood pressure, diastolic blood pressure, high density lipoprotein (HDL) cholesterol, triglycerides
\end{abstract}

\section{S. B. van Dijk $(\bowtie)$}

School of Clinical Health Sciences, Department of Physical

Therapy Science, Utrecht University,

Utrecht, the Netherlands

e-mail: s.b.vandijk@kpnmail.nl

T. Takken

Child Development \& Exercise Centre, Wilhelmina Children's Hospital, University Medical Centre Utrecht, Lundlaan 6, Room KB2.056.0, PO Box 85090, 3508 AB Utrecht, the Netherlands

E. C. Prinsen

Roessingh Research and Development,

Roessinghsbleekweg 33b, Room 16, PO Box 310, $7500 \mathrm{AH}$

Enschede, the Netherlands

H. Wittink

Research group Lifestyle and Health,

Research Centre for Innovation in Health Care,

University of Applied Sciences Utrecht,

Bolognalaan 101, Room 0.075, PO Box 85182, 3508 AD Utrecht, the Netherlands and fasting glucose. Two independent reviewers performed abstract, full text and data selection.

Results Twenty articles were included describing 21,618 males and 24,139 females. Waist circumference had the strongest correlation with all CVD risk factors for both men and women, except for HDL and LDL in men. When comparing BMI with waist circumference, the latter showed significantly better correlations to CVD risk factors, except for diastolic blood pressure in women and HDL and total cholesterol in men.

Conclusions We recommend the use of waist circumference in clinical and research studies above other anthropometric adiposity measures, especially compared with BMI, when evaluating CVD risk factors.

Keywords Meta-analysis · Cardiovascular disease risk factors - Anthropometric - Adiposity - Waist circumference

\section{Introduction}

The World Health Organisation reported cardiovascular disease (CVD) to be death cause number one globally with $29 \%$ of all-cause deaths, which is 17.1 million people, in 2004 [1]. Researchers found these mortality rates to be closely associated to certain CVD risk factors [2-4]. These CVD risk factors are abdominal obesity, high blood pressure, low high-density lipoprotein cholesterol (HDL-C) levels, high triglyceride levels and high fasting glucose [2]. The criteria of the Adult Treatment Panel III (ATP III) of the National Cholesterol Education Program state that having three out of these five CVD risk factors defines the metabolic syndrome and involves an overall increase in all-cause and CVD death [2-4].

Adiposity has proven to be an important risk factor for cardiovascular disease and is strongly associated with CVD 
risk factors [2, 5]. Most studies use anthropometric measures to measure adiposity. Waist circumference and waist-to-hip ratio have been used as measures of central obesity (where visceral adipose tissue is stored), and body mass index (BMI) $\left(\mathrm{kg} / \mathrm{m}^{2}\right)$ has been used as a measure of general obesity [6]. Studies that analysed associations between anthropometric measures and abdominal visceral fat, measured with computed tomography, reported waist circumference to be a better measure of central obesity [6-9].

A recent systematic review investigated the importance of obesity and cardiorespiratory fitness related to the risks of and mortality due to CVD and diabetes type 2 [5]. They found BMI to be the most frequently used measure of adiposity in the included studies and raise a few questions about the overall applicability of the BMI. For instance, BMI neither gives an indication of the relation between fat and fat-free mass, nor does it indicate fat distribution. For a given BMI, physically fit individuals have less total and abdominal fat, compared with unfit individuals. A large muscle mass instead of fat mass will also place people in higher BMI categories. This is supported by a large study that investigated the diagnostic accuracy of BMI to detect adiposity [10]. They found limited diagnostic performance of the BMI in correctly identifying individuals with excess in body fat (BF), particularly in those with $\mathrm{BMI}<30$. Although BMI has a good correlation with $\mathrm{BF} \%$, it failed to discriminate between $\mathrm{BF} \%$ and lean mass [10]. In addition, BMI is also criticised because it makes no difference between men and women, even though we know fat distribution is significantly different between men and women $[10,11]$. Also age and ethnicity have an effect on body fat distribution and are not differentiated in BMI [7, 11, 12]. Is it not wiser to use a different type of adiposity measurement?

Although relationships between anthropometric adiposity measures and CVD risk factors have been explored thoroughly in many studies around the world, results have to our knowledge never been combined quantitatively in a metaanalysis. To eliminate ethnic differences we choose to focus on studies that describe Caucasian populations.

Therefore the aim of this study was to study the following research question: which anthropometric adiposity measure has the strongest association with CVD risk factors in Caucasian men and women without a history of CVD?

\section{Methods}

Definitions

Cardiovascular disease risk factors

We chose to focus on the CVD risk factors reported by the ATP III definition [2]. High blood pressure (systolic blood pressure $>130 \mathrm{mmHg}$ and/or diastolic blood pressure
$>85 \mathrm{mmHg}$ ), low HDL-C (men $<1.03 \mathrm{mmol} / \mathrm{l}$; women $<1.30 \mathrm{mmol} / \mathrm{l})$, high triglycerides $(>1.70 \mathrm{mmol} / \mathrm{l})$ and fasting glucose $(>6.1 \mathrm{mmol} / \mathrm{l})$.

\section{Anthropometric adiposity measures}

We only considered simple measures that give an indication of fat mass or body fat distribution. The most frequently used measures were BMI, waist circumference, waist-to-hip ratio, waist-to-height ratio and $\mathrm{BF} \%$ calculated from skin folds or bio-electrical impedance. Studies that describe anthropometric methods not commonly used in practice were excluded. Examples of such methods are magnetic resonance imaging or dual-emission X-ray absorptiometry techniques.

\section{Body mass index}

General obesity is widely measured with the BMI. BMI is calculated as weight in $\mathrm{kg}$ divided by squared height in meters $\left(\mathrm{kg} / \mathrm{m}^{2}\right)$. There is consensus about the used cut-off points in a Caucasian population, which are described in Table $1[11,13]$.

\section{Waist circumference}

Waist circumference is reported as the better measurement for central obesity and therefore a good predictor of abdominal visceral fat [6-9]. There is general consensus about waist circumference cut-off points for increased CVD risk in a Caucasian population: $>102 \mathrm{~cm}$ for men and $>88 \mathrm{~cm}$ for women $[2,11,13]$. These cut-off points correspond to the BMI values for "obese class I" where $>90 \mathrm{~cm}$ for men and $>80 \mathrm{~cm}$ for women correspond to BMI values for "overweight/pre-obese" [14].

\section{Waist-to-hip ratio}

This ratio was developed because waist and hip circumferences measure different aspects of body composition and fat distribution and have independent and often opposite effects on CVD risk factors. A narrow waist and large hips
Table 1 BMI cut-off points

\begin{tabular}{ll}
\hline Classification & BMI $\left(\mathrm{kg} / \mathrm{m}^{2}\right)$ \\
\hline Underweight & $<18.5$ \\
Normal weight & $18.5-24.99$ \\
Overweight & $\geq 25.00$ \\
- Pre-obese & $25.00-29.99$ \\
- Obese I & $30.00-34.99$ \\
- Obese II & $35.00-39.99$ \\
- Obese III & $\geq 40.00$ \\
\hline
\end{tabular}


may both protect against cardiovascular disease [15]. Cutoff points that indicate increased CVD risk for waist-to-hip ratio have been calculated by Dobbelsteyn et al. and are 0.9 and 0.8 for men and women, respectively [14].

\section{Waist-to-height ratio}

This simple measure adds the factor height to the waist circumference. Like the other anthropometric adiposity measurements, ethnic differences require different cutoff points. For Caucasian populations, however, we did not find any well-established cut-off points for waist-toheight ratio.

\section{Body fat percentage}

Two methods of anthropometric BF\% measurement techniques have been developed and validated. First is the skin fold method where $\mathrm{BF} \%$ can be calculated from skin fold measures at four sites on the body [16]. Second bio-electrical impedance can be used to estimate $\mathrm{BF} \%$. With this method a small alternating current is applied to the body. Different body tissues have different resistivity and therefore a calculation can be made with this method to estimate the $\mathrm{BF} \%$ [17]. A BF\% of $>25 \%$ or $>35 \%$ for men and women, respectively, is correlated to a BMI of 30 and chosen as cut-off-points for Caucasian people [12].

\section{Caucasians}

Defining who is Caucasian or not is problematic. Strictly, they might be the people who lived in the Caucasus, but in common use a Caucasian is referred to as a European or someone with European ancestry. This is somewhat hard to control when examining a large population, for example, in the US or Australia. In scientific literature Caucasians are also sometimes called whites. In this review we chose to include only Caucasians because of reported differences in anthropometric adiposity measures and their associations with CVD risk factors in different ethnicities. In selecting articles for this review we chose to exclude mainly the studies that comprised populations of which we knew they differ from Caucasian populations. For example Asians [2, 12, 18, 19], Africans [2, 12, 20], Aboriginal Australians [21] and Hispanics/Latin Americans [2, 22] have been studied and reported to differ from standards based on Caucasian populations.

We included studies that describe European, American, Canadian and Australian populations. No studies were found that reported big differences between these populations. This does not mean, however, that differences do not exist.
Search strategy

A search strategy was created by the first author (SvD) with the support of a medical librarian and critically reviewed and approved by the direct supervisor (HW). After approval published articles in the following databases were searched: PubMed (1966-Nov. 2009), CINAHL (1966-Nov. 2009), EMBASE (1947-Nov. 2009) and Cochrane Controlled Trials Register (CENTRAL) (The Cochrane Library 2009 issue 11). The search strategy did not have any limitations on ethnicity. Five potentially eligible articles were manually searched before applying the search strategy. When conducting the search strategy these five articles had to be found to ensure that the strategy covered all our criteria. This method fine-tuned the strategy and created a small certainty that we did not miss important articles.

Eligibility criteria

\section{Types of participants}

Only studies that described results on Caucasian adults without a history of CVD were included.

\section{Types of studies}

All types of research designs were included. Only studies for which a full-text article in the English or Dutch language was available were considered for inclusion.

\section{Types of outcome measures}

Included were studies that reported correlations between different anthropometric adiposity measures and CVD risk factors. At least two anthropometric measures had to be compared with at least three of four CVD risk factors. Secondary outcomes were total cholesterol and lowdensity lipoprotein cholesterol data. The possibilities are displayed in Fig. 1. The study had to report correlation coefficients between anthropometric adiposity measures and CVD risk factors. If these correlations were not reported, but could be calculated from given data, the study was considered for inclusion. Correlation coefficients had to be reported for men and women separately and in the case of multi-ethnic studies the data had to be reported for each ethnic group, in order to extract Caucasian data. When this was not the case this study was excluded.

Study selection

One reviewer $(\mathrm{SvD})$ made a first selection of articles based on title only. Only study titles obviously not involving any study criteria for this review were excluded. 
Fig. 1 Graphical display of all outcome measures

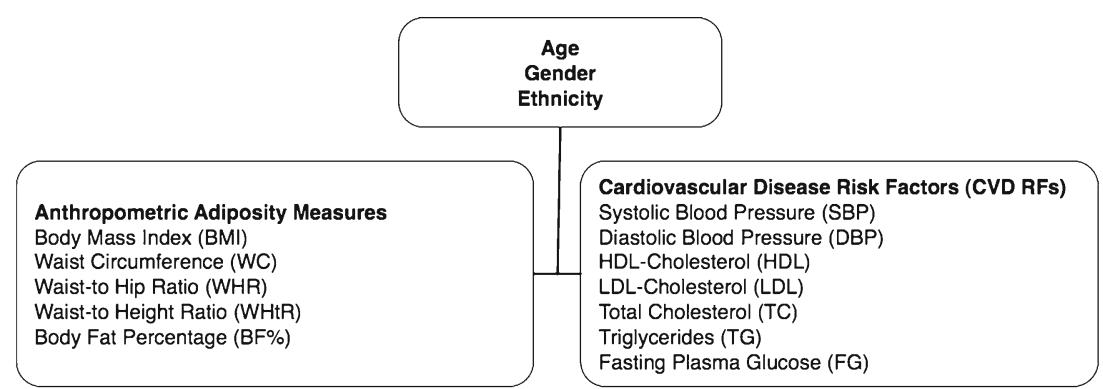

After exclusion based on title alone two independent reviewers (SvD and EP) selected articles based on the abstract. After selection, the reviewers independently applied the inclusion criteria on the full text articles and decided on inclusion or not. Disagreement with including articles was solved with discussion and a third reviewer was consulted if disagreement still persisted.

Study quality assessment

We chose not to assess methodological quality of the included studies. There is no consensus on which checklist to use and most checklists involve quality assessment of clinical trials [23]. Our research question can not be answered with results from clinical trials, so most available checklists will not be useful to us. Also we found no recommendations on how to incorporate a quality score in a meta-analysis.

\section{Data extraction}

In case of good but insufficient data presented in the studies, authors were mailed to gain more specific results that could be used in our meta-analysis. Data from included articles were summarised in a table. Two independent reviewers selected data on relevant characteristics to minimise potential bias or mistakes in the data extraction process. Characteristics described in the table were: first author and publication year, study name (acronym), baseline year(s), sample size, population age, population gender, population ethnicity, anthropometric adiposity measurements used, CVD risk factors assessed, association parameter used, statistical adjustments, association parameter results.

\section{Data interpretation}

Pearson correlations were interpreted according to magnitudes as proposed by Cohen et al. [24]. Very small: $0.0-0.1$, small: $0.1-0.3$, moderate: $0.3-0.5$, large: $0.5-0.7$, very large: $0.7-0.9$ and almost perfect: $0.9-1.0$.

\section{Statistical analyses}

We used a fixed-effects model for computing mean correlations. First, all correlations were converted to the Fisher's Z-scale and weighted by the number of subjects to calculate the mean Fisher's $Z$ as described by Field et al. in Eq. 2 [25]. These were inverted in the end to display an actual r-value. With use of standard deviations of these r-values and the number of studies performed, we calculated the $95 \%$ confidence interval $(95 \% \mathrm{CI})$ for each of the mean correlations and plotted the results for each CVD risk factor in a forest plot. By examining the $95 \% \mathrm{CI}$ we determined the statistical significance between the correlations for each of the anthropometric adiposity measures. When $95 \%$ CIs did not overlap we assumed a significant difference at the $p=0.05$ level. When $95 \%$ CIs overlapped, a $95 \%$ CI was calculated for the difference between the means and determined whether this $95 \% \mathrm{CI}$ contained zero. If it did contain zero the difference was not significant and if it did not contain zero the difference was deemed significant [26]. All statistical analyses were performed in Microsoft Office Excel 2008 for Mac.

\section{Results}

Study selection

The search strategy yielded a total of 991 hits. Ninety-eight articles initially appeared to meet inclusion criteria, 78 of which were eventually excluded, resulting in a total of 20 definite inclusions. A flowchart displaying exact details on the definite inclusion is presented in Fig. 2. Most exclusions in the full-text selection were made because complete data were not available, other statistics than Pearson or Spearman correlations were used, a separate analysis was not performed for ethnic groups or gender and there were multiple publications describing the same study population.

Study characteristics

The oldest article included was published in the year 1986 and the most recent study was published in 2009. Most 
Fig. 2 Article flow chart

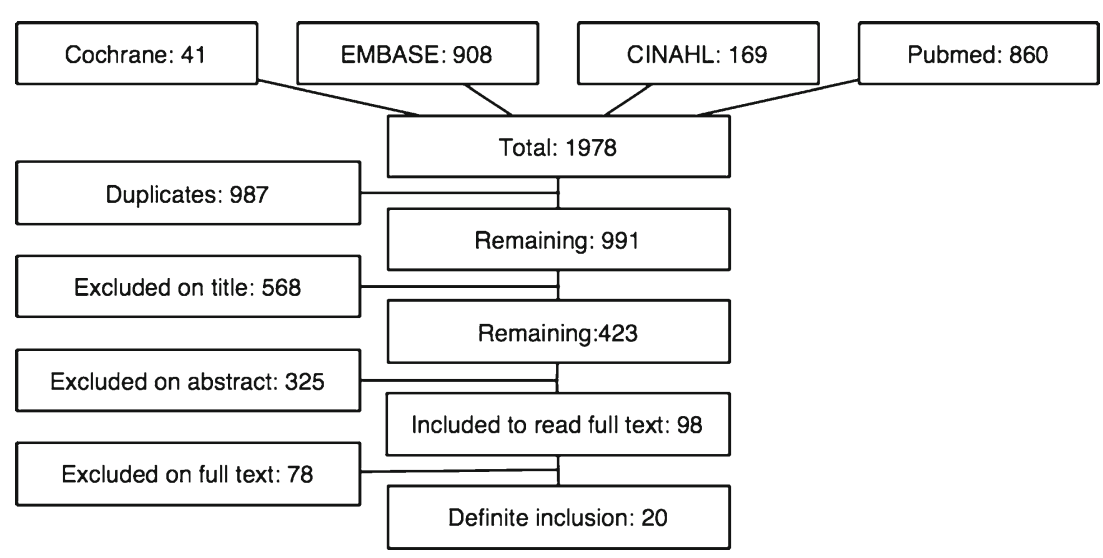

studies used cross-sectional designs and tested persons who voluntarily participated in preventive medical examinations. All studies together examined a total of 45,757 subjects; 21,618 males and 24,139 females. Nine studies, comprising $86 \%$ of all subjects together, included subjects roughly between 18 and 90 years of age. The other studies included only subjects within a smaller age spectrum.

Distribution of subjects in the four major regions was quite identical with 12,037 subjects from the USA (4 studies), 9820 subjects from Canada (3 studies), 11,247 subjects from Australia (1 study) and 12,653 subjects from Europe (12 studies).

BMI, waist circumference (WC), waist-to-hip ratio (WHR), waist-to-height ratio (WHtR) and $\mathrm{BF} \%$ were described in 19 , $16,17,6$ and 1 study respectively. $\mathrm{BF} \%$, calculated from four skin folds, was only reported by Contaldo et al. [27] and therefore there was no manner to quantitatively analyse this data. Results of this study were not included into our analysis.

Twelve studies described all four primary CVD outcome measures [15, 28-37], eight described only three [9, 38-44]. The study by Seidell et al. [41] described five different European populations and reported data for each population separately. These data were therefore analysed as five different study populations.

All studies used a Pearson correlation coefficient to determine associations except for the study by Ohrvall et al. [35] who used a Spearman rank correlation coefficient. Studies varied in use of adjustments in calculation of the correlation coefficients but when we studied the data we found no obvious differences between adjusted and unadjusted data. More detailed information about the study characteristics are displayed in Table 2.

Study results for men

All data on men are displayed in Fig. 3. Moderate correlations were found for all anthropometric adiposity measures with triglycerides (TG). Also, moderate correlations were found for WC with systolic blood pressure (SBP), and for
BMI and WC with diastolic blood pressure (DBP). All other correlations were small. We found the strongest correlations between WC and SBP, DBP, total cholesterol (TC), triglycerides (TG) and fasting glucose (FG). These correlations were significantly higher than those of other anthropometric adiposity measures for SBP and DBP. For HDL we found WC to correlate almost as well as BMI. For LDL we found WHR to have the best correlation, however not significant. We found WC to correlate significantly better than BMI with SBP, DBP, LDL, TG and FG. The weakest correlate to all the CVD risk factors was WHtR, significantly differing from other anthropometric adiposity measures for SBP, DBP, HDL, TC and FG.

\section{Study results for women}

All data on women are displayed in Fig. 4. Very small correlations were found for WHtR with LDL and TC. Moderate correlations were found for WC with SBP, WC and BMI with DBP, WC with HDL and BMI, WC and WHR with TG. All other correlations were small. For all CVD risk factors $\mathrm{WC}$ was the strongest correlate. This correlation was found to be statistically significant $(p<0.05)$ for SBP only. For LDL, TC, TG and FG, WHR approached the WC correlation, making WC not the overall significantly better correlate. WC correlates significantly better with all CVD risk factors than BMI $(p<0.05)$, except for DBP. We found $\mathrm{WHtR}$ to be the weakest correlate of all anthropometric adiposity measures.

\section{Discussion}

The aim of our study was to investigate which anthropometric adiposity measure had the strongest association with cardiovascular disease risk factors in Caucasian men and women without a history of cardiovascular disease. We found WC to have the strongest associations among almost all CVD risk factors for both men and women, although not always 


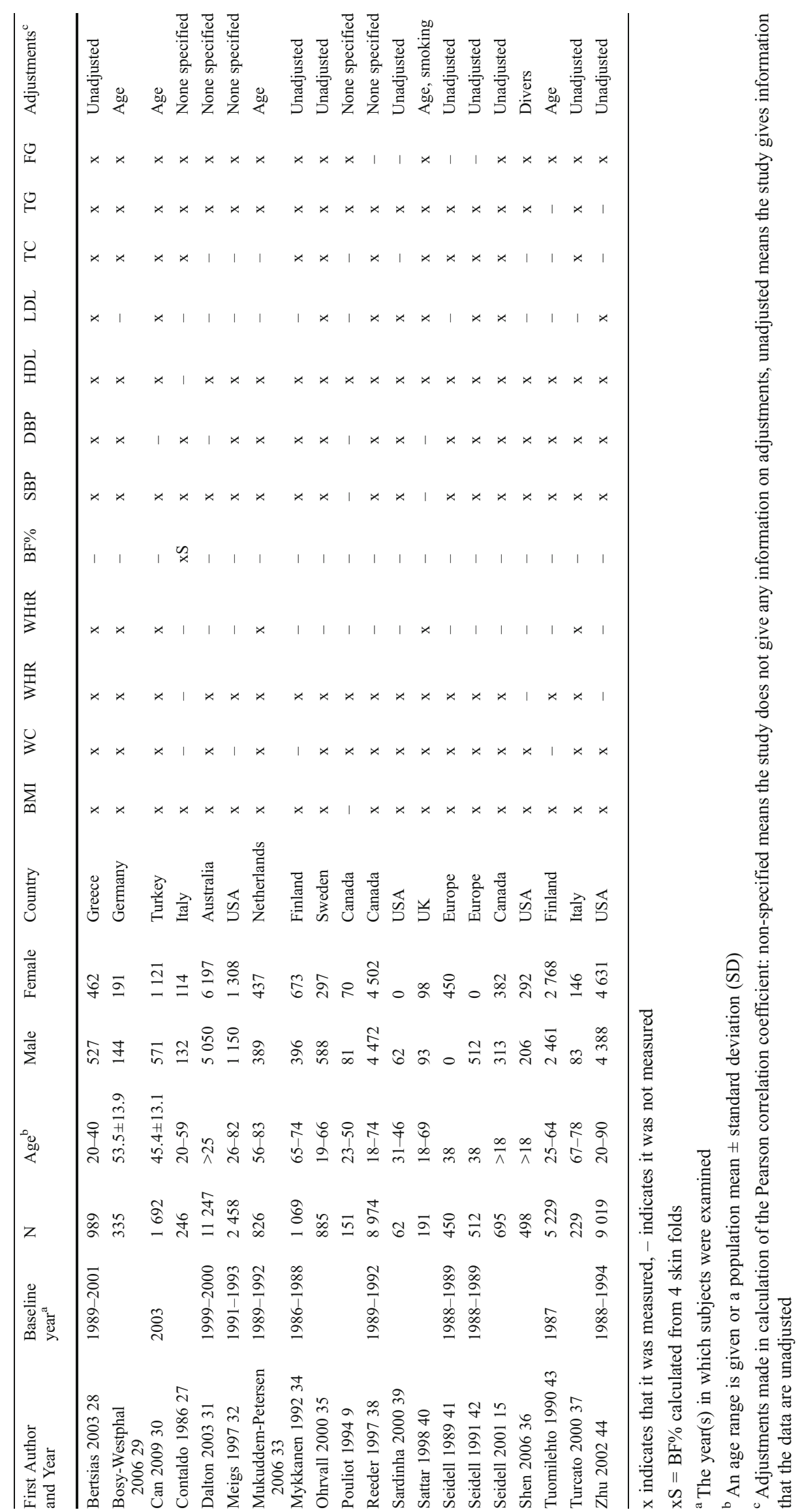




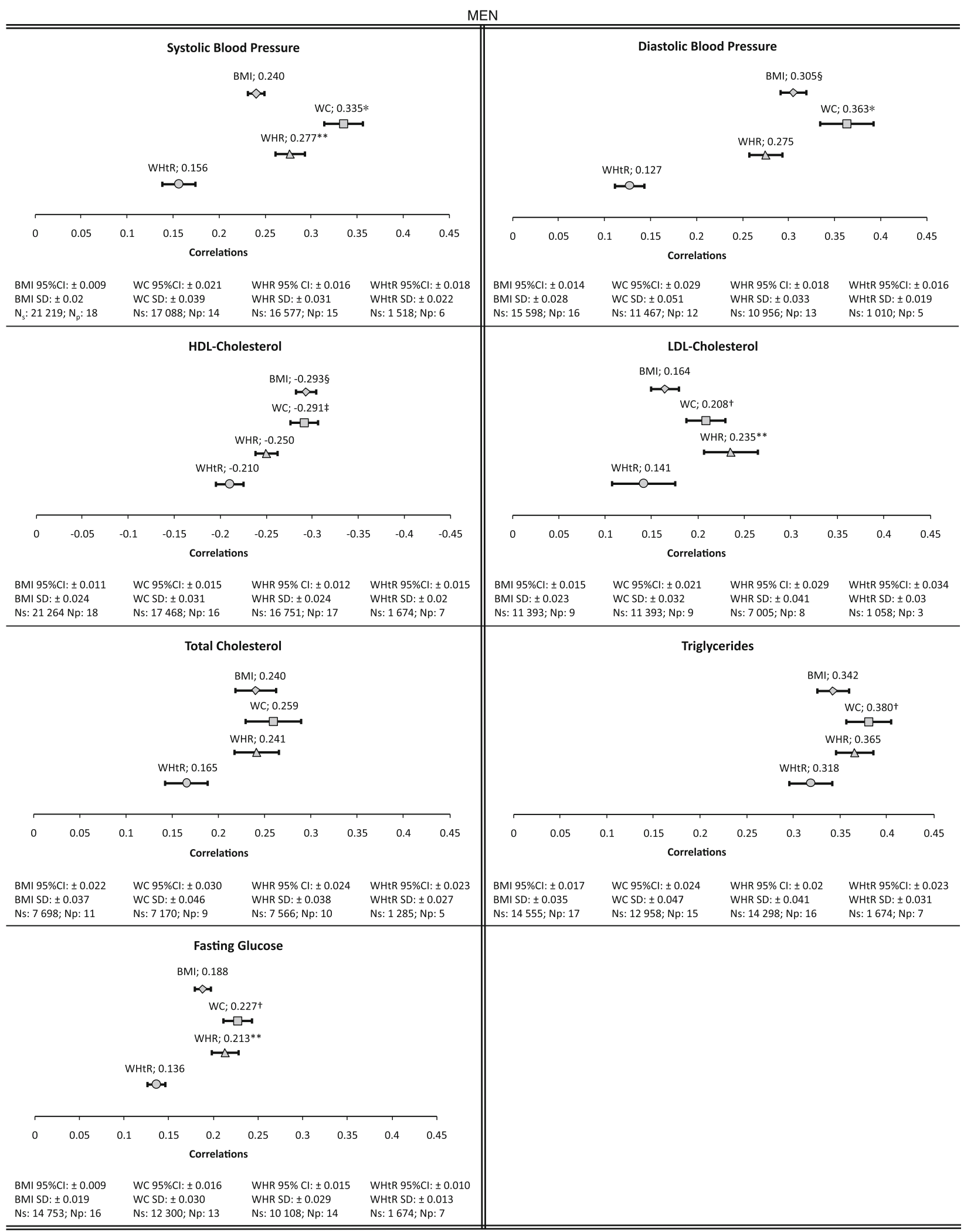

Fig. 3 Mean Pearson correlations and their 95\% confidence intervals plotted for men. $*$ WC correlates significantly better than BMI, WHR and WHtR $(P<0.05)$. $* *$ WHR correlates significantly better than BMI and WHtR $(P<0.05)$. †WC correlates significantly better than BMI and WHtR $(P<0.05)$. \$WC correlates significantly better than WHR and WHtR $(P<0.05)$. $\S$ BMI correlates significantly better than WHR and WHtR $(P<0.05) . S D$ standard deviation; Ns number of subjects; $\mathrm{Np}$ number of populations 


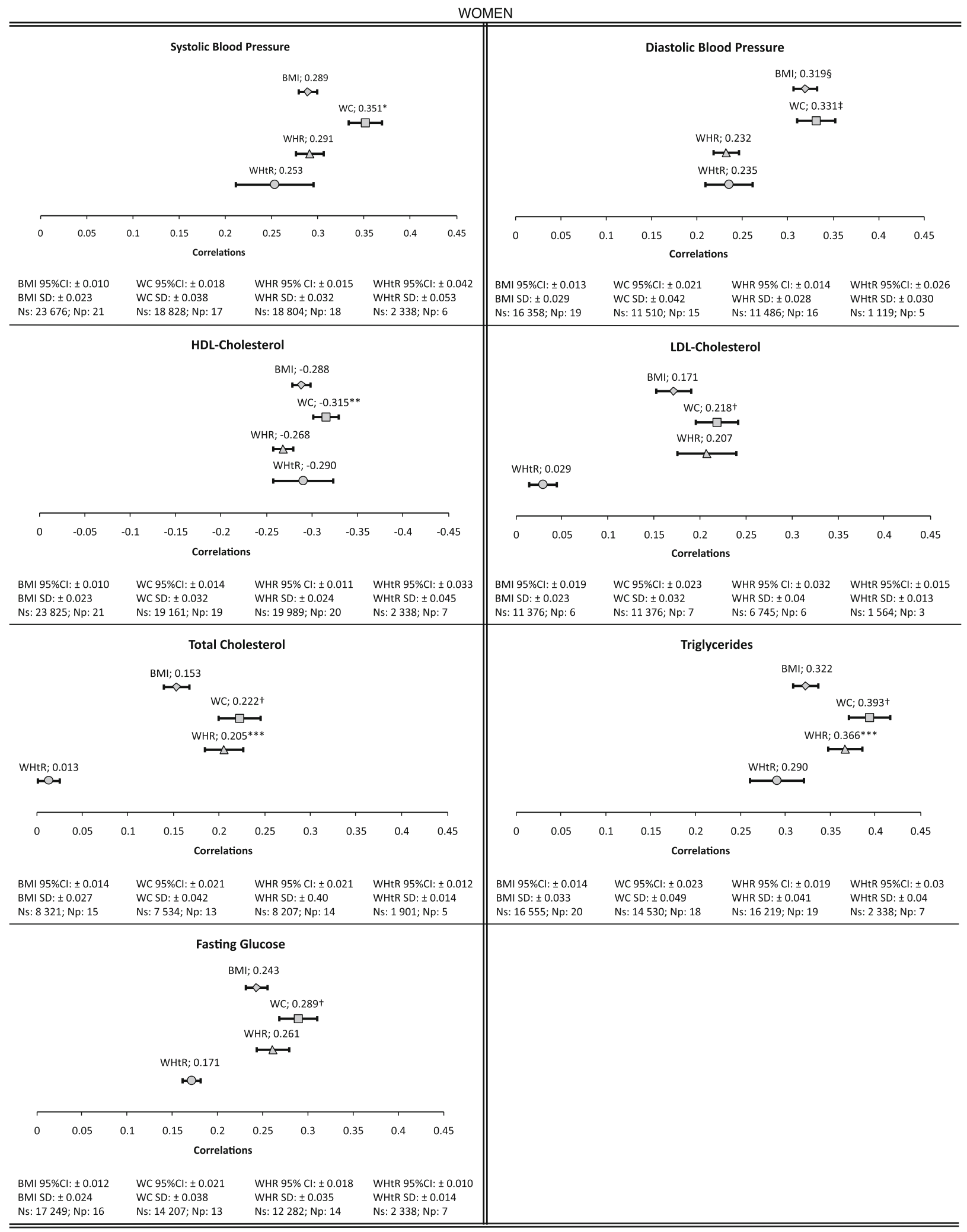

Fig. 4 Mean Pearson correlations and their 95\% confidence intervals plotted. $*$ WC correlates significantly better than BMI, WHR and WHtR $(P<0.05) . * *$ WC correlates significantly better than BMI and WHR $(P<0.05) . * * *$ WHR correlates significantly better than BMI and WHtR $(P<0.05)$. $†$ WC correlates significantly better than BMI and
WHtR $(P<0.05)$. \$WC correlates significantly better than WHR and WHtR $(P<0.05)$. §BMI correlates significantly better than WHR and WHtR $(P<0.05) . S D$ standard deviation; Ns number of subjects; $N p$ number of populations 
significantly. Especially when BMI was compared with WC the latter holds significantly better correlations to CVD risk factors except for DBP in women and HDL and TC in men. Comparing BMI and WHR resulted in only small and mostly non-significant differences except for TC and TG in women and LDL in men in favour of WHR. The weakest correlations were found for WHtR.

Our study selection procedure yielded only one study that used a measure of BF\% [27]. This study reported rather moderate and large correlations between $\mathrm{BF} \%$ and $\mathrm{CVD}$ risk factors $(0.54,0.4,0.37,0.37$ and 0.36 with SBP, DBP, TC, $\mathrm{TG}$ and FG respectively for women, $0.38,0.46,0.37,0.42$ and 0.31 with SBP, DBP, TC, TG and FG respectively for men). This study compared $\mathrm{BF} \%$ with $\mathrm{BMI}$ and also found moderate and large correlations between BMI and CVD risk factors. Based on these data, $\mathrm{BF} \%$ could be a competitor for the strongest correlations with CVD risk factors; however, more studies should be done before any conclusions can be drawn.

Janssen et al. [45] analysed the data from the NHANES III database to determine whether the prevalence of CVD risk factors is greater in individuals with high WC values compared with individuals with normal WC values within the same BMI category. Individuals with high WC values were reported to have significantly greater prevalence of CVD risk factors even within the same BMI category, thus underscoring the importance of WC. When evaluating the clustering of CVD risk factors in the metabolic syndrome, Schneider et al. [46] and Dervaux et al. [47] concluded that WC had a stronger association with the metabolic syndrome than BMI.

One should also consider some practical issues with anthropometric adiposity measures. The general idea behind using anthropometric adiposity measures is to predict a certain risk and to measure change over time when comparing interventions. The ability to detect change in the different anthropometric adiposity measures can play an important role here. Velthuis et al. [48] conducted a randomised controlled trial and investigated the effect of a 12month moderate-to-vigorous exercise program combining aerobic and muscle strength training on body composition among 189 sedentary, postmenopausal women. Their data showed that the exercise program was able to reduce fat mass, increase lean body mass and reduce WC, although weight and BMI were not affected. Kwak et al. [49] found similar results in a randomised controlled trial with 553 male and female subjects. This further supports the use of alternative anthropometric adiposity measures next to BMI, such as WC, as a more responsive outcome.

This meta-analysis holds a few study limitations. Heterogeneity of study populations remains a subject of discussion. Whether Europeans differ amongst each other and can be compared with for example Canadians is not known. We found no studies describing objective differences among these populations and in order to reach sufficient power in our analysis we made the choice to compare all these populations. Although the subject inclusion criteria for all studies were almost the same it remains difficult to control for other known CVD risk factors, for example smoking status, physical activity or fitness levels, social status and nutrition.

Studies might have been missed by our search method even though we tried to validate the strategy. Studies describing associations between only one anthropometric adiposity measure and three or less CVD risk factors were not included although maybe holding valuable data for our analysis. Furthermore, a few large studies found with our strategy did not report correlation coefficients and were therefore excluded after attempting to get the data from authors by mail and failing. Calculating the needed correlation coefficients would be easily done and could alter or strengthen the results of our study.

A comment should also be made about the fact that we studied CVD risk factors and not cardiovascular diseases or mortality rates. As we found correlations between anthropometric adiposity measures and CVD risk factors were generally small to moderate, it can be expected that even weaker correlations with actual cardiovascular disease will be found. This was addressed in a recent large study performed by The Emerging Risk Factors Collaboration [50]. They concluded that BMI, WC, and WHR, whether assessed singly or in combination, did not importantly improve cardiovascular disease risk prediction in people in developed countries when additional information is available for systolic blood pressure, history of diabetes, and lipids [50].

Although relationships between anthropometric adiposity measures and CVD risk factors have been explored thoroughly in many studies around the world this is the first study to our knowledge to combine these results quantitatively in a meta-analysis. Waist circumference has been a widely used measure for adiposity for some time now and we believe the evidence supporting its use has been strengthened with the current study. The results of our study can, however, only be generalised to people of Caucasian descent.

\section{Recommendations}

Although overall correlations between CVD risk factors and anthropometric adiposity measures were small, they do appear to be significant. Given the extent of the cardiovascular disease problem in the industrialised world, it is important clinicians should use a measure that most accurately reflects CVD risk. From this study we concluded that the measurement of WC was more related to CVD risk factors in men and 
women than BMI. We therefore recommend the use of WC in the clinic and in research studies.

\section{Conflict of interest None declared.}

Open Access This article is distributed under the terms of the Creative Commons Attribution Noncommercial License which permits any noncommercial use, distribution, and reproduction in any medium, provided the original author(s) and source are credited.

\section{References}

1. WHO. http://www.who.int/mediacentre/factsheets/fs317/en/index. html. In.

2. ATPIII. Third report of the National Cholesterol Education Program (NCEP) Expert Panel on Detection, Evaluation, and Treatment of High Blood Cholesterol in Adults (Adult Treatment Panel III). Final Report 2002, Bethesda, Maryland, USA: National Institutes of Health.

3. Ford ES. Risks for all-cause mortality, cardiovascular disease, and diabetes associated with the metabolic syndrome: a summary of the evidence. Diabetes Care. 2005;28(7):1769-78.

4. Galassi A, Reynolds K, He J. Metabolic syndrome and risk of cardiovascular disease: a meta-analysis. Am J Med. 2006;119 (10):812-9.

5. Fogelholm M. Physical activity, fitness and fatness: relations to mortality, morbidity and disease risk factors. A systematic review. Obes Rev. 2009.

6. Molarius A, Seidell JC. Selection of anthropometric indicators for classification of abdominal fatness - a critical review. Int J Obes Relat Metab Disord. 1998;22(8):719-27.

7. Hill JO, Sidney S, Lewis CE, et al. Racial differences in amounts of visceral adipose tissue in young adults: the CARDIA (Coronary Artery Risk Development in Young Adults) study. Am J Clin Nutr. 1999;69(3):381-7.

8. Vazquez G, Duval S, Jacobs Jr DR, et al. Comparison of body mass index, waist circumference, and waist/hip ratio in predicting incident diabetes: a meta-analysis. Epidemiol Rev. 2007;29:11528.

9. Pouliot MC, Despres JP, Lemieux S, et al. Waist circumference and abdominal sagittal diameter: best simple anthropometric indexes of abdominal visceral adipose tissue accumulation and related cardiovascular risk in men and women. Am J Cardiol. 1994;73(7):460-8.

10. Romero-Corral A, Somers VK, Sierra-Johnson J, et al. Accuracy of body mass index in diagnosing obesity in the adult general population. Int J Obes (Lond). 2008;32(6):959-66.

11. WHO. World Health Organisation. Obesity: preventing and managing the global epidemic. Report of a WHO consultation. World Health Organ Tech Rep Ser. 2000;894: i-xii, 1-253.

12. Deurenberg P, Yap M, van Staveren WA. Body mass index and percent body fat: a meta analysis among different ethnic groups. Int J Obes Relat Metab Disord. 1998;22(12):1164-71.

13. NIH. The practical guide identification, evaluation, and treatment of over-weight and obesity in adults. Bethesda, MD: National Institutes of Health, 2000. (NIH publication no. 00-4084.).

14. Dobbelsteyn CJ, Joffres MR, MacLean DR, et al. A comparative evaluation of waist circumference, waist-to-hip ratio and body mass index as indicators of cardiovascular risk factors. The Canadian Heart Health Surveys. Int J Obes Relat Metab Disord. 2001;25 (5):652-61.
15. Seidell JC, Perusse L, Despres JP, et al. Waist and hip circumferences have independent and opposite effects on cardiovascular disease risk factors: the Quebec Family Study. Am J Clin Nutr. 2001;74(3):315-21.

16. Durnin JV, Womersley J. Body fat assessed from total body density and its estimation from skinfold thickness: measurements on 481 men and women aged from 16 to 72 years. Br J Nutr. 1974;32 (1):77-97.

17. Jaffrin MY. Body composition determination by bioimpedance: an update. Curr Opin Clin Nutr Metab Care. 2009;12(5):482-6.

18. Park SH, Choi SJ, Lee KS, et al. Waist circumference and waist-toheight ratio as predictors of cardiovascular disease risk in Korean adults. Circ J. 2009;73(9):1643-50.

19. Bhopal R, Unwin N, White M, et al. Heterogeneity of coronary heart disease risk factors in Indian, Pakistani, Bangladeshi, and European origin populations: cross sectional study. BMJ. 1999;319 (7204):215-20.

20. Craig P, Colagiuri S, Hussain Z, et al. Identifying cut-points in anthropometric indexes for predicting previously undiagnosed diabetes and cardiovascular risk factors in the Tongan population. Obesity Research and Clinical Practice. 2007;1(1):17-25.

21. Piers LS, Rowley KG, Soares MJ, et al. Relation of adiposity and body fat distribution to body mass index in Australians of Aboriginal and European ancestry. Eur J Clin Nutr. 2003;57(8):956-63.

22. Sundquist J, Winkleby MA. Cardiovascular risk factors in Mexican American adults: a transcultural analysis of NHANES III, 1988-1994. Am J Public Health. 1999;89(5):723-30.

23. Moja LP, Telaro E, D'Amico R, et al. Assessment of methodological quality of primary studies by systematic reviews: results of the metaquality cross sectional study. BMJ. 2005;330(7499):1053-5.

24. Cohen J. Statistical power analysis for the behavioral sciences. 2nd ed. New Jersey: Erlbaum; 1988.

25. Field AP. Meta-analysis of correlation coefficients: a Monte Carlo comparison of fixed- and random-effects methods. Psychol Methods. $2001 ; 6(2): 161-80$.

26. Knezevic A. Overlapping confidence intervals and statistical significance (http://www.cscu.cornell.edu/news/statnews/stnews73. pdf). In: Cornell University, Cornell Statistical Consulting Unit, 2008.

27. Contaldo F, Di Biase G, Panico S, et al. Body fat distribution and cardiovascular risk in middle-aged people in southern Italy. Atherosclerosis. 1986;61(2):169-72.

28. Bertsias G, Mammas I, Linardakis M, et al. Overweight and obesity in relation to cardiovascular disease risk factors among medical students in Crete, Greece. BMC Publ Health. 2003;3:1-9.

29. Bosy-Westphal A, Danielzik S, Geisler C, et al. Use of height3: waist circumference 3 as an index for metabolic risk assessment? Br J Nutr. 2006;95(6):1212-20.

30. Can AS, Bersot TP, Gonen M. Anthropometric indices and their relationship with cardiometabolic risk factors in a sample of Turkish adults. Public Health Nutrition. 2009;12(4):538-46.

31. Dalton M, Cameron AJ, Zimmet PZ, et al. Waist circumference, waist-hip ratio and body mass index and their correlation with cardiovascular disease risk factors in Australian adults. Journal of Internal Medicine. 2003;254(6):555-63.

32. Meigs JB, D'Agostino Sr RB, Wilson PWF, et al. Risk variable clustering in the insulin resistance syndrome: the Framingham Offspring Study. Diabetes. 1997;46(10):1594-600.

33. Mukuddem-Petersen J, Snijder MB, et al. Sagittal abdominal diameter: no advantage compared with other anthropometric measures as a correlate of components of the metabolic syndrome in elderly from the Hoorn Study. Am J Clin Nutr. 2006;84(5):9951002.

34. Mykkanen L, Laakso M, Pyorala K. Association of obesity and distribution of obesity with glucose tolerance and cardiovascular risk factors in the elderly. Int J Obes. 1992;16(9):695-704. 
35. Ohrvall M, Berglund L, Vessby B. Sagittal abdominal diameter compared with other anthropometric measurements in relation to cardiovascular risk. Int J Obes. 2000;24(4):497-501.

36. Shen W, Punyanitya M, Chen J, et al. Waist circumference correlates with metabolic syndrome indicators better than percentage fat. Obesity (Silver Spring). 2006;14(4):727-36.

37. Turcato E, Bosello O, Di Francesco V, et al. Waist circumference and abdominal sagittal diameter as surrogates of body fat distribution in the elderly: their relation with cardiovascular risk factors. Int J Obes Relat Metab Disord. 2000;24(8):1005-10.

38. Reeder BA, Senthilselvan A, Despres JP, et al. The association of cardiovascular disease risk factors with abdominal obesity in Canada. Canadian Medical Association Journal. 1997;157 Suppl 1:S39-45.

39. Sardinha LB, Teixeira PJ, Guedes DP, et al. Subcutaneous central fat is associated with cardiovascular risk factors in men independently of total fatness and fitness. Metabolism. 2000;49(11):1379-85.

40. Sattar N, Tan CE, Han TS, et al. Associations of indices of adiposity with atherogenic lipoprotein subfractions. Int $\mathrm{J}$ Obes. 1998;22(5):432-9.

41. Seidell JC, Cigolini M, Charzewska J, et al. Indicators of fat distribution, serum lipids, and blood pressure in European women born in 1948 - the European Fat Distribution Study. Am J Epidemiol. 1989;130(1):53-65.

42. Seidell JC, Cigolini M, Deslypere JP, et al. Body fat distribution in relation to serum lipids and blood pressure in 38-year-old European men: the European fat distribution study. Atherosclerosis. 1991;86 (2-3):251-60.
43. Tuomilehto J, Marti B, Kartovaara L, et al. Body fat distribution, serum lipoproteins and blood pressure in middle-aged Finnish men and women. Rev Epidemiol Sante Publique. 1990;38(5-6):507-15.

44. Zhu K, Wang Z, Heshka S, et al. Waist circumference and obesityassociated risk factors among whites in the third National Health and Nutrition Examination Survey: clinical action thresholds. Am J Clin Nutr. 2002;76(4):743-9.

45. Janssen I, Katzmarzyk PT, Ross R. Body mass index, waist circumference, and health risk: evidence in support of current National Institutes of Health guidelines. Arch Intern Med. 2002;162 (18):2074-9.

46. Schneider HJ, Glaesmer H, Klotsche J, et al. Accuracy of anthropometric indicators of obesity to predict cardiovascular risk. J Clin Endocrinol Metab. 2007;92(2):589-94.

47. Dervaux N, Wubuli M, Megnien JL, et al. Comparative associations of adiposity measures with cardiometabolic risk burden in asymptomatic subjects. Atherosclerosis. 2008;201(2):413-7.

48. Velthuis MJ, Schuit AJ, Peeters PH, et al. Exercise program affects body composition but not weight in postmenopausal women. Menopause. 2009;16(4):777-84.

49. Kwak L, Kremers SP, Candel MJ, et al. Changes in skinfold thickness and waist circumference after 12 and 24 months resulting from the NHF-NRG In Balance-project. Int J Behav Nutr Phys Act. 2010;7(1):26.

50. Wormser D, Kaptoge S, Di Angelantonio E, et al. Separate and combined associations of body-mass index and abdominal adiposity with cardiovascular disease: collaborative analysis of 58 prospective studies. Lancet. 2011;377(9771):1085-95. 